\title{
PROPERTIES OF HIGH-DENSITY MATTER IN NEUTRON STARS
}

\author{
Fridolin Weber * \\ Department of Physics, San Diego State University, \\ 5500 Campanile Drive, San Diego, California 92182, USA and \\ Center for Astrophysics and Space Sciences, \\ University of California, \\ San Diego, La Jolla, California 92093, USA. \\ Gustavo A. Contrerađ \\ Department of Physics, San Diego State University, \\ 5500 Campanile Drive, San Diego, California 92182, USA, \\ CONICET, Rivadavia 1917, 1033 Buenos Aires, Argentina, \\ IFLP, CONICET - Dpto. de Física, UNLP, La Plata, Argentina and \\ Gravitation, Astrophysics and Cosmology Group, \\ Facultad de Ciencias Astronómicas y Geofísicas, UNLP, \\ Paseo del Bosque S/N (1900), La Plata, Argentina. \\ Milva G. Orsaria \\ Department of Physics, San Diego State University, \\ 5500 Campanile Drive, San Diego, California 92182, USA, \\ CONICET, Rivadavia 1917, 1033 Buenos Aires, Argentina and \\ Gravitation, Astrophysics and Cosmology Group, \\ Facultad de Ciencias Astronómicas y Geofísicas, UNLP, \\ Paseo del Bosque S/N (1900), La Plata, Argentina. \\ William Spinella $\$$ and Omair Zubairi \\ Department of Physics $\&$ Computational Science Researech Center, \\ San Diego State University, 5500 Campanile Drive, San Diego, California 92182, USA.
}

(Dated: August 4, 2014)

\begin{abstract}
This short review aims at giving a brief overview of the various states of matter that have been suggested to exist in the ultra-dense centers of neutron stars. Particular emphasis is put on the role of quark deconfinement in neutron stars and on the possible existence of compact stars made of absolutely stable strange quark matter (strange stars). Astrophysical phenomena, which distinguish neutron stars from quark stars, are discussed and the question of whether or not quark deconfinement may occur in neutron stars is investigated. Combined with observed astrophysical data, such studies are invaluable to delineate the complex structure of compressed baryonic matter and to put firm constraints on the largely unknown equation of state of such matter.
\end{abstract}

\section{INTRODUCTION}

Exploring the properties of compressed baryonic matter, or, more generally, strongly interacting matter at high densities and/or temperatures, has become a forefront area of modern physics. Experimentally, the properties of such matter are being probed with the Relativistic Heavy Ion Collider RHIC at Brookhaven and the Large Hadron Collider (LHC at CERN). Great advances in our understanding of such matter are expected from the next generation of heavyion collision experiments at FAIR (Facility for Antiproton and Ion Research at GSI) and NICA (Nuclotron-bases Ion Collider fAcility at JINR) [1-3].

Neutron stars contain compressed baryonic matter permanently in their centers. Such stars are remnants of massive stars that blew apart in core-collapse supernova explosions. They are typically about 20 kilometers across and spin rapidly, often making many hundred rotations per second. Many neutron stars form radio pulsars, emitting radio

\footnotetext{
*Electronic address: fweber@mail.sdsu.edu

${ }^{\dagger}$ Electronic address: contrera@fisica.unlp.edu.ar

${ }^{\ddagger}$ Electronic address: morsaria@rohan.sdsu.edu

§Electronic address: spinellla@mail.sdsu.edu

IElectronic address: zubairi@rohan.sdsu.edu
} 
waves that appear from the Earth to pulse on and off like a lighthouse beacon as the star rotates at very high

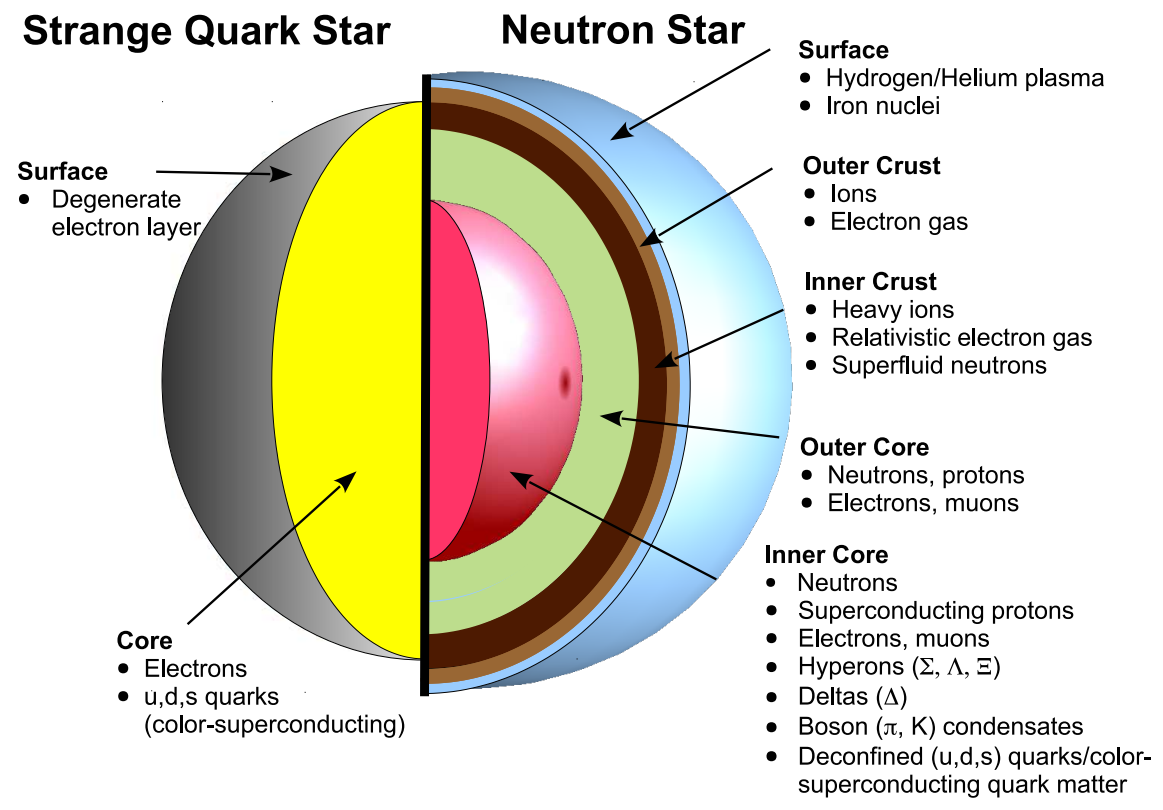

Figure 1: (Color online) Schematic structures of quark stars (left) and neutron stars (right). If the strange quark matter hypothesis should be correct, most, if not all, neutron stars should in fact be strange quark stars.

speeds. Neutron stars in X-ray binaries accrete material from a companion star and flare to life with tremendous bursts of X-rays. Depending on mass and rotational frequency, gravity compresses the matter in the cores of neutron stars to densities that are several times higher than the density of ordinary atomic nuclei. At such huge densities atoms themselves collapse, and atomic nuclei are squeezed so tightly together that new fundamental particles may be produced and novel states of matter be created. The most spectacular phenomena stretch from the generation of hyperons and delta particles, to the formation of meson condensates, to the formation of a plasma of deconfined quarks (see Fig. 1). The interest in the role of quark deconfinement for astrophysics has received renewed interest by the discovery that quark matter at low temperatures ought to be a color superconductor (see Ref. [4] and references therein).

All these features make neutron stars superb astrophysical laboratories for a wide range of physical studies 5 - 12 . And with observational data accumulating rapidly from both orbiting and ground based observatories spanning the spectrum from X-rays to radio wavelengths, there has never been a more exiting time than today to study neutron stars and associated catastrophic astrophysical events. The Hubble Space Telescope and X-ray satellites such as Chandra and XMM-Newton in particular have proven especially valuable. New astrophysical instruments such as the Five hundred meter Aperture Spherical Telescope (FAST), the square kilometer Array (skA), Fermi Gamma-ray Space Telescope (formerly GLAST), Astrosat, ATHENA (Advanced Telescope for High ENergy Astrophysics), and the Neutron Star Interior Composition Explorer (NICER) promise the discovery of tens of thousands of new neutron stars. Of particular interest will be the proposed NICER mission (scheduled to launch in 2016), which is dedicated to the study of extraordinary gravitational, electromagnetic, and nuclear-physics environments embodied by neutron stars. NICER will explore the exotic states of matter in the core regions of neutron stars, where, as mentioned just above, density and pressure are considerably higher than in atomic nuclei, confronting nuclear theory with unique observational constraints.

There is also the theoretical possibility that quark matter made of up, down and strange quarks (so-called strange quark matter [13]) may be more stable than ordinary nuclear matter [14]. This so-called strange matter hypothesis constitutes one of the most startling possibilities regarding the behavior of superdense matter, which, if true, would have implications of fundamental importance for cosmology, the early universe, its evolution to the present day, and astrophysical compact objects such as neutron stars and white dwarfs (see Ref. [15] and references therein). The properties of compact stars made of strange quark matter, referred to as strange (quark) stars, are compared with those of neutron stars in Table \and Fig. 1. Even after three decades of research, there is no sound scientific basis on 
Table I: Theoretical properties of strange quark stars and neutron stars compared.

\begin{tabular}{ll}
\hline \hline Strange Quark Stars & Neutron Stars \\
\hline Made entirely of deconfined up, down, strange & Nucleons, hyperons, boson condensates, \\
$\quad$ quarks, and electrons & deconfined quarks, electrons, and muons \\
Absent & Superfluid neutrons \\
Absent & Superconducting protons \\
Color superconducting quarks & Color superconducting quarks \\
Energy per baryon $\lesssim 930 \mathrm{MeV}$ & Energy per baryon $>930 \mathrm{MeV}$ \\
Self-bound $\left(M \propto R^{3}\right)$ & Bound by gravity \\
Maximum mass $\sim 2 M_{\odot}$ & Same \\
No minimum mass if bare & Minimum mass $\sim 0.1 M_{\odot}$ \\
Radii $R \lesssim 10-12 \mathrm{~km}$ & Radii $R \gtrsim 10-12 \mathrm{~km}$ \\
Baryon numbers $B \lesssim 10^{57}$ & Baryon numbers $10^{56} \lesssim B \lesssim 10^{57}$ \\
Electric surface fields $\sim 10^{18}$ to $\sim 10^{19} \mathrm{~V} / \mathrm{cm}$ & Absent \\
Can either be bare or enveloped in thin & Always have nuclear crusts \\
nuclear crusts $\left(\right.$ masses $\left.\lesssim 10^{-5} M_{\odot}\right)$ & \\
Maximum density of crust set by neutron drip, i.e., & Does not apply, i.e., neutron stars \\
strange stars posses only outer crusts & posses inner and outer crusts \\
Form two-parameter stellar sequences & Form one-parameter stellar sequences \\
\hline \hline
\end{tabular}

which one can either confirm or reject the strange quark matter hypothesis so that it remains a serious possibility of fundamental significance for various astrophysical phenomena, as discussed in the next section [16].

\section{PROPERTIES OF STRANGE QUARK STARS}

A bare quark star differs qualitatively from a neutron star which has a density at the surface of about 0.1 to $1 \mathrm{~g} / \mathrm{cm}^{3}$. The thickness of the quark surface is just $\sim 1 \mathrm{fm}$, the length scale of the strong interaction. The electrons at the surface of a quark star are held to quark matter electrostatically, and the thickness of the electron surface is several hundred fermis. Since neither component, electrons and quark matter, is held in place gravitationally, the Eddington limit to the luminosity that a static surface may emit does not apply, so that bare quark stars may have photon luminosities much greater than $10^{38} \mathrm{erg} / \mathrm{s}$. It has been shown in Ref. [17] that this value may be exceeded by many orders of magnitude by the luminosity of $e^{+} e^{-}$pairs produced by the Coulomb barrier at the surface of a hot strange star. For a surface temperature of $\sim 10^{11} \mathrm{~K}$, the luminosity in the outflowing pair plasma was calculated to be as high as $\sim 3 \times 10^{51} \mathrm{erg} / \mathrm{s}$. Such an effect may be a good observational signature of bare strange stars [17 20]. If the strange star is dressed, that is, enveloped in a nuclear crust, however, the surface made of ordinary atomic matter would be subject to the Eddington limit. Hence the photon emissivity of a dressed quark star would be the same as for an ordinary neutron star. If quark matter at the stellar surface is in the CFL (Color-Flavor Locked) phase the process of $e^{+} e^{-}$pair creation at the stellar quark matter surface may be turned off. This may be different for the early stages of a very hot CFL quark star [21].

In contrast to neutron stars, the radii of self-bound quark stars decrease the lighter the stars, according to $M \propto R^{3}$. The existence of nuclear crusts on quark stars changes the situation drastically [15, 22]. Since the crust is bound gravitationally, the mass-radius relationship of quark stars with crusts can be qualitatively similar to mass-radius relationships of neutron stars and white dwarfs [22]. In general, quark stars with or without nuclear crusts possess smaller radii than neutron stars. This implies that quark stars have smaller mass shedding (break-up) periods than neutron stars. Moreover, due to the smaller radii of quarks stars, the complete sequence of quark stars-and not just those close to the mass peak, as it is the case for neutron stars-can sustain extremely rapid rotation [15, 22]. In particular, a strange star with a typical pulsar mass of around $1.45 M_{\odot}$ has a Kepler period in the approximate range of $0.55 \lesssim P_{\mathrm{K}} / \mathrm{msec} \lesssim 0.8$ [22, 23]. This is to be compared with $P_{\mathrm{K}} \sim 1 \mathrm{msec}$ for neutron stars of the same mass.

One of the most amazing features of strange quark stars concerns the existence of ultra-high electric fields on their surfaces, which, for ordinary (i.e., non-superconducting) quark matter, is around $10^{18} \mathrm{~V} / \mathrm{cm}$. If strange matter forms a color superconductor, as expected for such matter, the strength of the electric field may increase to values that exceed $10^{19} \mathrm{~V} / \mathrm{cm}$. The energy density associated with such huge electric fields is on the same order of magnitude as the energy density of strange matter itself, which may alter the masses and radii of strange quark stars at the $15 \%$ 
and $5 \%$ level, respectively [24].

The electrons at the surface of a quark star are not necessarily in a fixed position but may rotate with respect to the quark matter star [25]. In this event magnetic fields can be generated which, for moderate effective rotational frequencies between the electron layer and the stellar body, agree with the magnetic fields inferred for several Central Compact Objects (CCOs). These objects could thus be interpreted as quark stars whose electron atmospheres rotate at frequencies that are moderately different $(\sim 10 \mathrm{~Hz})$ from the rotational frequency of the quark star itself.

Last but not least, we mention that the electron surface layer may be strongly affected by the magnetic field of a quark star in such a way that the electron layer performs vortex hydrodynamical oscillations [26]. The frequency spectrum of these oscillations has been derived in analytic form in Ref. [26]. If the thermal X-ray spectra of quark stars are modulated by vortex hydrodynamical oscillations, the thermal spectra of compact stars, foremost central compact objects (CCOs) and X-ray dim isolated neutron stars (XDINSs), could be used to verify the existence of these vibrational modes observationally. The central compact object 1E 1207.4-5209 appears particularly interesting in this context, since its absorption features at $0.7 \mathrm{keV}$ and $1.4 \mathrm{keV}$ can be comfortably explained in the framework of the hydro-cyclotron oscillation model 26 ].

Rotating superconducting quark stars ought to be threaded with rotational vortex lines, within which the star's interior magnetic field is at least partially confined. The vortices (and thus magnetic flux) would be expelled from the star during stellar spin-down, leading to magnetic re-connection at the surface of the star and the prolific production of thermal energy. It has been shown in Ref. 27] that this energy release can re-heat quark stars to exceptionally high temperatures, such as observed for Soft Gamma Repeaters (SGRs), Anomalous X-Ray pulsars (AXPs), and X-ray dim isolated neutron stars (XDINs), and that SGRs, AXPs, and XDINs may be linked ancestrally [27].

The conversion of a neutron star to a hypothetical quark star could lead to quark novae. Such events could explain gamma ray bursts [28], the production of heavy elements such as platinum through r-process nucleosynthesis [29], and double-humped super-luminous supernovae [30].

\section{NEUTRON STARS}

\section{A. Non-spherical neutron stars}

Usually, the structure of neutron stars is modeled with the assumption that they are perfect spheres. However, due to to very high magnetic fields, certain classes of neutron stars, such as magnetars and neutron stars containing cores of color superconducting quark matter [31], may be deformed. The stellar structure equation of such stars is given by 32 ]

$$
\frac{d P}{d r}=-(\epsilon+P)\left(\frac{1}{2} r+4 \pi r^{3} P-\frac{1}{2} r\left(1-\frac{2 m}{r}\right)^{\gamma}\right)\left(r^{2}\left(1-\frac{2 m}{r}\right)^{\gamma}\right)^{-1} .
$$

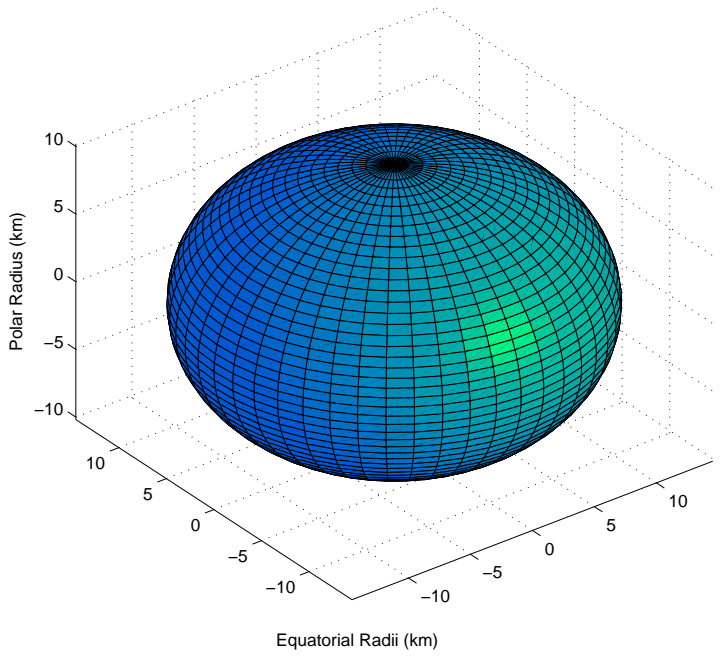

Figure 2: Oblate spheroid $(\gamma=0.70)$

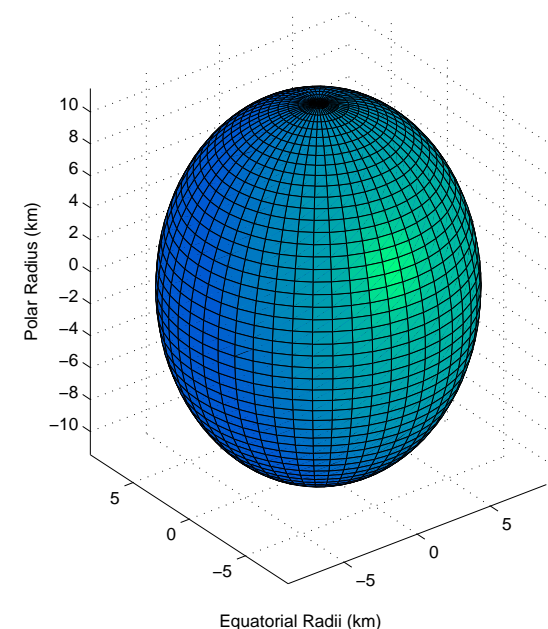

Figure 3: Prolate spheroid $(\gamma=1.30)$ 


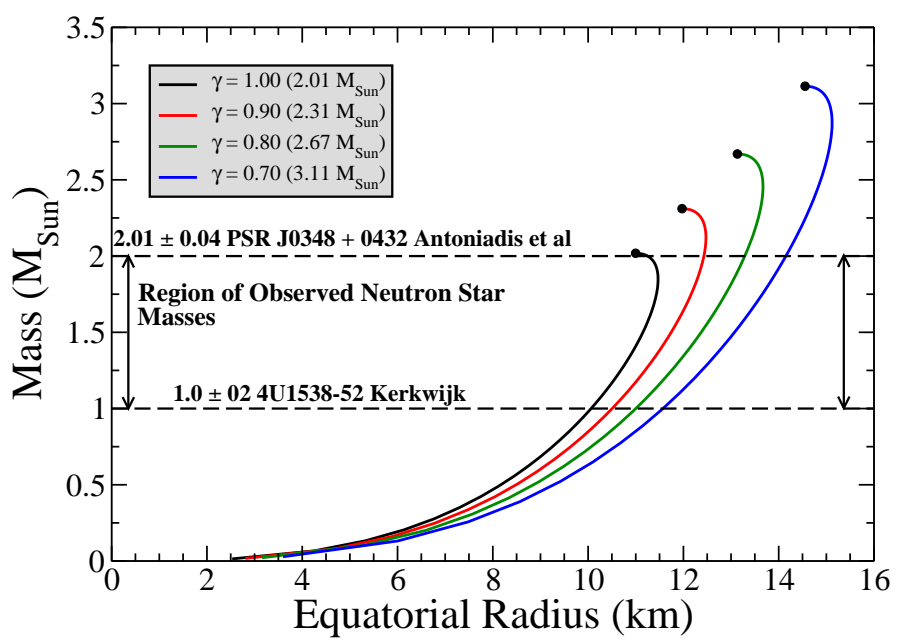

Figure 4: (Color online) Mass-radius relationships of sequences of oblate strange quark stars. The mass is increasing with increasing oblateness (decreasing values of $\gamma$ ). The range of observed neutron star masses is indicated.

Here, $\epsilon$ is the energy-density, $P$ is the pressure, $m$ is the gravitational mass, and $\gamma$ is a deformation constant. The star's total mass is given by $M=\gamma m$. Oblate (prolate) neutron stars are obtained for $\gamma<1(\gamma>1)$, as shown in Figs. 2] and 3, respectively. For $\gamma=1$, equation (11) reduces to the well known Tolman-Oppenheimer-Volkoff equation, which describes the properties of spherically symmetric neutron (compact) stars [5, 6, 15].

The mass-radius relationship of oblate compact stars, computed from Eq. (11) for different $\gamma$ values, are shown in Fig. 目

For simplicity, the equation of state of a relativistic gas of deconfined quarks described by the MIT bag model, $P=(\epsilon-4 B) / 3$, has been used here [32]. The value of the bag constant is $B=57 \mathrm{MeV} / \mathrm{fm}^{3}\left(B^{1 / 4}=145 \mathrm{MeV}\right)$, which makes the quark gas absolutely stable with respect to nuclear matter. The results shown in Figs. 2 to 4 therefore are for strange quark stars. One sees that already relatively small deviations from spherical symmetry increase the masses of strange stars substantially.

\section{B. Rotation-driven compositional changes}

The change in central density of a neutron star whose frequency varies from zero to the mass shedding (Kepler) frequency can be as large as 50 to $60 \%$ [15]. This suggests that changes in the rotation rate of a neutron star may drive phase transitions and/or lead to significant compositional changes in the star's core [15, 33, 34]. As a case in point, for some rotating neutron stars the mass and initial rotational frequency may be just such that the central density rises from below to above the critical density for dissolution of baryons into their quark constituents. This may be accompanied by a sudden shrinkage of the neutron star, effecting the star's moment of inertia and, thus, its spin-down behavior. As shown in Ref. [35], the spin-down of such a neutron star may be stopped or even reversed for tens of thousands to hundreds of thousands of years [15, 35]. The observation of an isolated neutron star which is spinning-up, rather than down, could thus hint at the existence of quark matter in its core.

\section{Quark deconfinement in high-mass neutron stars}

Quark deconfinement in high-mass neutron stars has very recently been studied using extensions of the local and non-local 3-flavor Nambu-Jona Lasinio (NJL) model supplemented with repulsive vector interactions among the quarks (see Refs. [36, 37] and references therein). The phase transition from confined hadronic matter to deconfined quark matter has been constructed via the Gibbs condition, which imposes global rather than local electric charge neutrality and baryon number conservation. Depending on the strength of the quark vector repulsion, it was found that an extended mixed phase of confined hadronic matter and deconfined quarks can exist in neutron stars as massive as 2.1 to $2.4 M_{\odot}$. A phase of pure quark matter inside such high-mass neutron stars, while not excluded, is only obtained for certain parametrizations of the underlying lagrangians. The radii of all these stars are between 12 and $13 \mathrm{~km}$, as expected for neutron stars of that mass [38 [40]. 

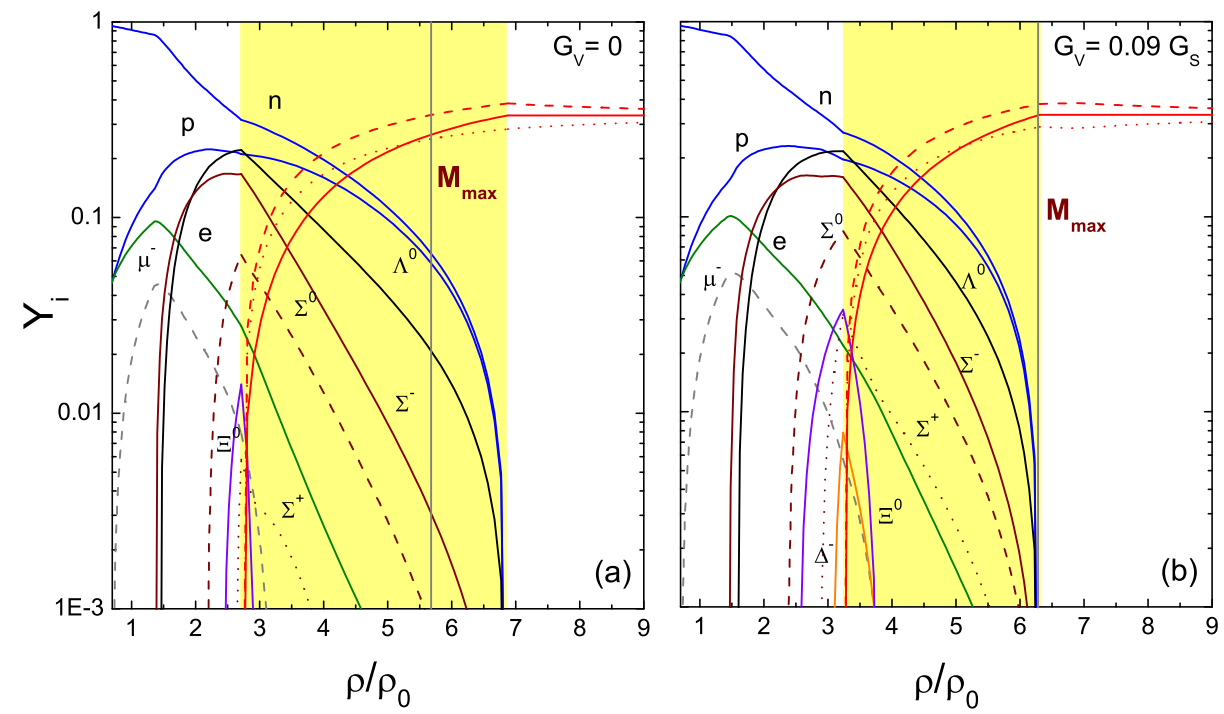

Figure 5: (Color online) Particle population of neutron star matter computed for the non-local SU(3) NJL model. The yellow areas highlight the mixed phase. The solid vertical lines indicate the central densities of the associated maximum-mass NSs. The hadronic model parametrization is NL3 and the vector repulsions are (a) $G_{V} / G_{S}=0$ and (b) $G_{V} / G_{S}=0.09$.

In Fig. 5 we show the relative particle fractions $Y_{i}\left(\equiv \rho_{i} / \rho\right)$ of neutron star matter as a function of baryon number density for the non-local NJL model and using NL3 parametrization of Ref. [41] for the hadronic model. It can be seen that by increasing the strength of the vector interaction, negatively charged particles like $\mu^{-}$'s and $\Delta^{-}$'s take on the role of electrons, whose primary duty is to make the stellar matter electrically neutral. Because of the early onset of the $\Delta$ population in this model, there is less need for electrons so that their number density in the mixed phase is
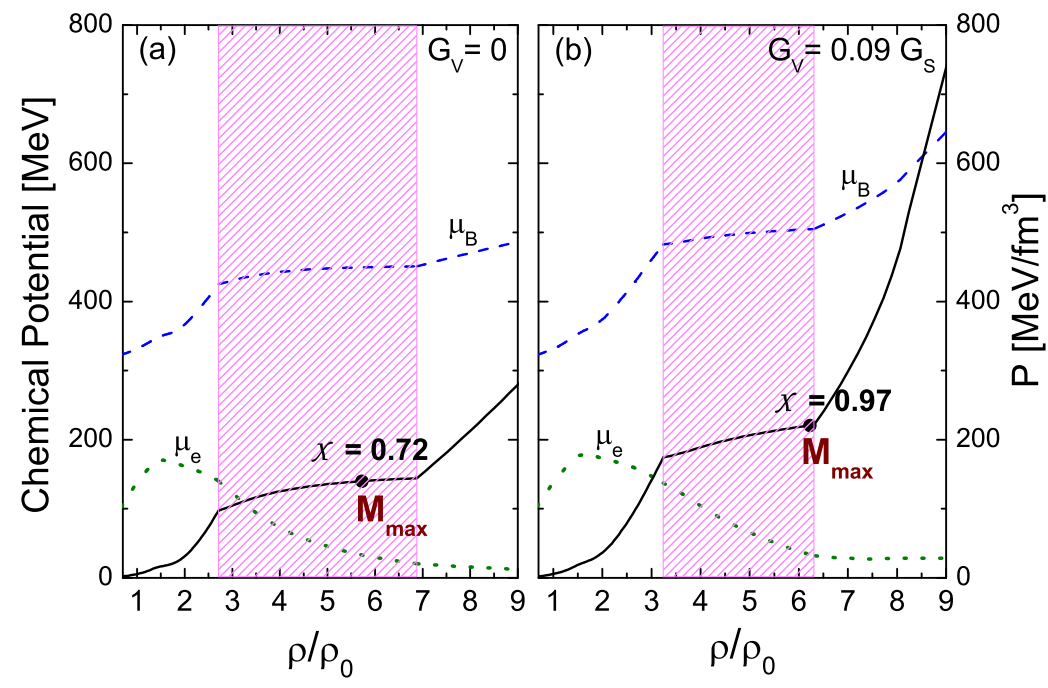

Figure 6: (Color online) Pressure $P$ (solid lines), baryon chemical potential $\mu_{B}=\mu_{n} / 3$ (dashed lines), and electron chemical potential $\mu_{e}$ (dotted lines) as a function of baryon number density (in units of $\rho_{0}=0.16 \mathrm{fm}^{-3}$ ). In all the cases a non local SU(3) NJL and non linear Walecka (with parametrization NL3) models are considered for quark matter and hadronic phases, respectively. The hatched areas denote the mixed phase regions where confined hadronic matter and deconfined quark matter coexist. Panel (a) is computed for zero vector repulsion. The impact of finite values of the vector repulsion $\left(G_{V}=0.09 G_{S}\right)$ on the data is shown in panel (b).

reduced compared to the outcome of standard mean-field/bag model calculations. The corresponding deleptonization densities, i.e. the densities beyond which leptons are no longer present in quark-hybrid matter, depend on the ratio $G_{V} / G_{S}\left(\rho^{G_{V}=0}=4.55 \rho_{0}\right.$ and $\left.\rho^{G_{V}=0.09 G_{S}}=5.33 \rho_{0}\right)$ 37]. 
Since we model the quark-hadron phase transition in three-space, accounting for the fact that the electric and baryonic charge are conserved for neutron star matter, the pressure varies monotonically with the proportion of the phases in equilibrium, as shown in Fig. 6. The hatched areas shown in this figure denote the mixed phase regions where confined hadronic matter and deconfined quark matter coexist. The quark matter contents of the maximummass neutron stars computed for these equations of state are also indicated ( $\chi$ values). Pure quark matter would exist for $\chi=1$. Our calculations show that, in the non-local case, the inclusion of the quark vector coupling contribution shifts the onset of the phase transition to higher densities and narrows the width of the mixed quark-hadron phase, when compared to the case $G_{V}=0$. To the contrary, when the quark matter phase is represented by the local NJL model, the width of the mixed phase tends to be broader for finite $G_{V} / G_{S}$ values 37 ].

To account for the uncertainty in the theoretical predictions of the ratio $G_{V} / G_{S}$, we treat the vector coupling constant as a free parameter. We observed that the non local NJL model is more sensitive to the increase of $G_{V} / G_{S}$ than the local model. For $G_{V} / G_{S}>0.09$ we have a shift of the onset of the quark-hadron phase transition to higher and higher densities, preventing quark deconfinement in the cores of neutron stars. However, we can reach values of $G_{V} / G_{S}$ up to 0.3 for the local NJL case.

\section{D. "Backbending"-a possible signal of quark deconfinement}

Whether or not quark deconfinement exists in static (non-rotating) neutron stars makes only very little difference to their properties, such as the range of possible masses and radii, which renders the detection of quark matter in such objects extremely complicated. This may be strikingly different for rotating neutron stars which develop quark matter cores in the course of spin-down. The reason being that such stars become more and more compressed as they spin down from high to low frequencies. For some rotating neutron stars the mass and initial rotational frequency may be just such that the central density rises from below to above the critical density for the dissolution of baryons into their quark constituents. This could affect the star's moment of inertia dramatically [15, 35]. Depending on the rate at which quark matter is produced, the moment of inertia can decrease very anomalously, and could even introduce an era of stellar spin-up (so-called "backbending") lasting for $\sim 10^{8}$ years [35]. Since the dipole age of millisecond pulsars is about $10^{9}$ years, one may estimate that roughly about $10 \%$ of the solitary millisecond pulsars presently known could be in the quark transition epoch and thus could be signaling the ongoing process of quark deconfinement. Changes in the moment of inertia reflect themselves in the braking index, $n$, of a rotating neutron star, as can be seen from [15, 35, 42]

$$
n(\Omega) \equiv \frac{\Omega \ddot{\Omega}}{\dot{\Omega}^{2}}=3-\frac{I+3 I^{\prime} \Omega+I^{\prime \prime} \Omega^{2}}{I+I^{\prime} \Omega} \rightarrow 3-\frac{3 I^{\prime} \Omega+I^{\prime \prime} \Omega^{2}}{2 I+I^{\prime} \Omega}
$$

where dots and primes denote derivatives with respect to time and $\Omega$, respectively. The last relation in Eq. (2) constitutes the non-relativistic limit of the braking index. It is obvious that these expressions reduce to the canonical limit, $n=3$, if the moment of inertia is completely independent of frequency. Evidently, this is not the case for rapidly rotating neutron stars, and it fails for stars that experience pronounced internal changes (as possibly driven by phase transitions) which alter the moment of inertia significantly. In Ref. [43] it was shown that the changes in the moment of inertia caused by the gradual transformation of hadronic matter into quark matter may lead to $n(\Omega) \rightarrow \pm \infty$ at the transition frequency where pure quark matter is produced. Such dramatic anomalies in $n(\Omega)$ are not known for conventional neutron stars (see, however, Ref. [4] ), because their moments of inertia appear to vary smoothly with $\Omega$ [6]. The future astrophysical observation of a strong anomaly in the braking behavior of a pulsar may thus indicate that quark deconfinement is occurring at the pulsar's center.

\section{E. Quark-hadron Coulomb lattices in the cores of neutron stars}

Because of the competition between the Coulomb and the surface energies associated with the positively charged regions of nuclear matter and negatively charged regions of quark matter, the mixed phase may develop geometrical structures (e.g., blobs, rods, slabs, as schematically illustrated in Fig. 7 [45]), similarly to what is expected of the subnuclear liquid-gas phase transition 46 49]. The consequences of such a Coulomb lattice for the thermal and transport properties of neutron stars have been studied in Ref. [45]. It was found that at low temperatures of $T \lesssim 10^{8} \mathrm{~K}$ the neutrino emissivity from electron-blob Bremsstrahlung scattering is at least as important as the total contribution from all other Bremsstrahlung processes (such as nucleon-nucleon and quark-quark Bremsstrahlung) and modified nucleon and quark Urca processes. It is also worth noting that the scattering of degenerate electrons off rare phase 


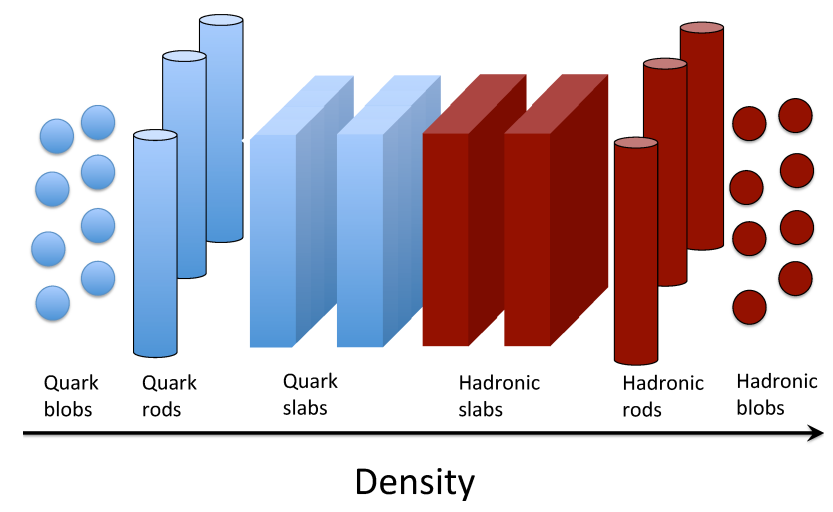

Figure 7: Schematic illustration of possible geometrical structures in the quark-hadron mixed phase of neutron stars. The structures may form because of the competition between the Coulomb and the surface energies associated with the positively charged regions of nuclear matter and negatively charged regions of quark matter.

blobs in the mixed phase region lowers the thermal conductivity by several orders of magnitude compared to a quarkhadron phase without geometric patterns. This may lead to significant changes in the thermal evolution of the neutron stars containing solid quark-hadron cores, which has not yet been studied.

\section{F. Pycnonuclear reaction rates}

The presence of strange quark nuggets in the crustal matter of neutron stars could be a consequence of Witten's strange quark matter hypothesis [14]. The impact of such nuggets on the pycnonuclear reaction rates among heavy atomic nuclei has been studied in Ref. [50]. Particular emphasis was put on the consequences of color superconductivity on the reaction rates. Depending on whether or not quark nuggets are in a color superconducting state, their electric charge distributions differ drastically, which was found to have dramatic consequences for the pycnonuclear reaction rates in the crusts of neutron stars. Future nuclear fusion network calculations may thus have the potential to shed light on the existence of strange quark matter nuggets and on whether they are in a color superconducting state, as suggested by QCD.

\section{G. Rotational instabilities}

The r-mode instability of a rotating neutron star dissipates the star's rotational energy by coupling the angular momentum of the star to gravitational waves $[51$ 53. This instability can be active in a newly formed isolated neutron star as well as in old neutron stars being spun up by accretion of matter from binary stars. If the interior contains quark matter, the r-mode instability and the gravitational wave signal may carry information about quark matter [54 58].

\section{Acknowledgments}

F. W. is supported by the National Science Foundation (USA) under Grants PHY-0854699 and PHY-1411708. M. O. and G. C. acknowledge CONICET and SeCyT-UNLP (Argentina) for financial support. M. O. and G. C. are thankful for hospitality extended to them during their visits at the SDSU, supported by a NSF-CONICET International Cooperation Project.

[1] The CBM Physics Book, B. Friman, C. Höhne, J. Knoll, S. Leupold, J. Randrup, R. Rapp, and P. Senger (Eds.), Lecture Notes in Physics 814 (Springer, 2011).

[2] NICA White Paper, http://nica.jinr.ru/files/WhitePaper.pdf.

[3] T. Klähn, D. Blaschke, and F. Weber, Phys. Part. Nucl. Lett. 9, 484 (2012). 
[4] M. G. Alford, A. Schmitt, K. Rajagopal, \& T. Schäfer, Rev. Mod. Phys. 13, 1455 (2008).

[5] N. K. Glendenning, Compact Stars, Nuclear Physics, Particle Physics, and General Relativity, 2nd ed. (Springer-Verlag, New York, 2000).

[6] F. Weber, Pulsars as Astrophysical Laboratories for Nuclear and Particle Physics, High Energy Physics, Cosmology and Gravitation Series (IOP Publishing, Bristol, Great Britain, 1999).

[7] F. Weber, Prog. Part. Nucl. Phys. 54, 193 (2005).

[8] J. M. Lattimer and M. Prakash, ApJ 550, 426 (2001).

[9] Physics of Neutron Star Interiors, D. Blaschke, N. K. Glendenning, and A. Sedrakian (Eds.), Lecture Notes in Physics $\mathbf{5 7 8}$ (Springer, 2001).

[10] A. Sedrakian, Prog. Part. Nucl. Phys. 58, 168 (2007).

[11] D. Page and S. Reddy, Ann. Rev. Nucl. Part. Sci. 56, 327 (2006).

[12] T. Hell and W. Weise, arXiv:1402.4098 [nucl-th].

[13] E. Farhi and R. L. Jaffe Phys. Rev. D 30, 2379 (1984).

[14] E. Witten, Phys. Rev. D 30, 272 (1984).

[15] F. Weber, Prog. Part. Nucl. Phys., 54, 193 (2005).

[16] M. Buballa et al., EMMI Rapid Reaction Task Force Meeting on "Quark Matter in Compact Star", arXiv:1402.6911 [astro-ph.HE].

[17] V. V. Usov, Phys. Rev. Lett. 80, 230 (1998).

[18] V. V. Usov, ApJ 550, L179 (2001).

[19] V. V. Usov, ApJ 559, L137 (2001).

[20] K. S. Cheng and T. Harko, ApJ 596, 451 (2003).

[21] C. Vogt, R. Rapp and R. Ouyed, Nucl. Phys. A 735, 543 (2004).

[22] N. K. Glendenning, Ch. Kettner, and F. Weber, ApJ 450, 253 (1995).

[23] N. K. Glendenning and F. Weber, ApJ 400, 647 (1992).

[24] R. Negreiros, F. Weber, M. Malheiro, and V. Usov, Phys. Rev. D 80, 083006 (2009).

[25] R. Negreiros, I. N. Mishustin, S. Schramm, and F. Weber, Phys. Rev. D 82, 103010 (2010).

[26] R. X. Xu, S. I. Bastrukov, F. Weber, J. W. Yu, and I. V. Molodtsova, Phys. Rev. D 85, 023008 (2012).

[27] B. Niebergal, R. Ouyed, R. Negreiros, and F. Weber, Phys. Rev. D 81, 043005 (2010).

[28] J. Staff, B. Niebergal, and R. Ouyed, MNRAS 391, 178 (2008).

[29] P. Jaikumar, B. S. Meyer, K. Otsuki and R. Ouyed, A\&A 471, 227 (2007).

[30] R. Ouyed and D. Leahy, A\&A 13, 1202 (2013).

[31] E. J. Ferrer et al., Phys. Rev. C 82, 065802 (2010).

[32] O. Zubairi Non-spherical Models of Neutron Stars, thesis proposal, San Diego State University, 2014.

[33] F. Weber, R. Negreiros, P. Rosenfield, and M. Stejner, Prog. Part. Nucl. Phys. 59, 94 (2007).

[34] R. Negreiros, S. Schramm, and F. Weber, Phys. Lett. B 718, 1176 (2013).

[35] N. K. Glendenning, S. Pei, and F. Weber, Phys. Rev. Lett. 79, 1603 (1997).

[36] M. Orsaria, H. Rodrigues, F. Weber and G. A. Contrera, Phys. Rev. D 87, 023001 (2013).

[37] M. Orsaria, H. Rodrigues, F. Weber and G. A. Contrera, Phys. Rev. C 89, 015806 (2014).

[38] A. W. Steiner, J. M. Lattimer and E. F. Brown, ApJ 722, 33 (2010).

[39] A. W. Steiner, J. M. Lattimer and E. F. Brown, ApJ 765, L5 (2013).

[40] J. M. Lattimer and A. W. Steiner, ApJ 784, 123 (2014).

[41] G. A. Lalazissis, J. Konig, and P. Ring, Phys. Rev. C 55, 540 (1997).

[42] N. K. Spyrou and N. Stergioulas, A\&A 395, 151 (2002).

[43] N. K. Glendenning and S. Pei, Phys. Rev. C 52, 2250 (1995).

[44] J. L. Zdunik, P. Haensel and M. Bejger, A $\& A$ 441, 207 (2005).

[45] X. Na, R. Xu, F. Weber, and R. Negreiros, Phys. Rev. D 86, 123016 (2012).

[46] D. G. Ravenhall, C. J. Pethick, and J. R. Wilson, Phys. Rev. Lett. 50, 2066 (1983).

[47] D. G. Ravenhall, C. J. Pethick, and J. M. Lattimer, Nucl. Phys. A 407, 571 (1983).

[48] R. D. Williams and S. E. Koonin, Nucl. Phys. A 435, 844 (1985).

[49] N. K. Glendenning, Phys. Rev. D 46, 1274 (1992).

[50] B. Golf, J. Hellmers, and F. Weber, Phys. Rev. C 80, 015804 (2009).

[51] N. Andersson, ApJ 502, 708 (1998).

[52] L. Lindblom, B. J. Owen and S. M. Morsink, Phys. Rev. Lett. 80, 4843 (1998).

[53] J. L. Friedman and S. M. Morsink, ApJ 502, 714 (1998).

[54] P. Jaikumar, G. Rupak and A. W. Steiner, Phys. Rev. D 78, 123007 (2008).

[55] G. Rupak and P. Jaikumar, Phys. Rev. C 82055806 (2010).

[56] G. Rupak and P. Jaikumar, Phys. Rev. C 88, 065801 (2013).

[57] B. A. Sa'd, arXiv:0806.3359 [astro-ph].

[58] M. Mannarelli, C. Manuel and B. A. Sa'd, Phys. Rev. Lett. 101, 241101 (2008). 